\title{
Insertion of $\beta$-satellite repeats identifies a transmembrane protease causing both congenital and childhood onset autosomal recessive deafness
}

\author{
Hamish S. Scott ${ }^{1,6}$, Jun Kudoh ${ }^{2}$, Marie Wattenhofer ${ }^{1}$, Kazunori Shibuya ${ }^{2}$, Asher Berry ${ }^{3}$, Roman Chrast ${ }^{1}$, \\ Michel Guipponi ${ }^{1}$, Jun Wang ${ }^{2}$, Kazuhiko Kawasaki ${ }^{2}$, Shuichi Asakawa ${ }^{2}$, Shinsei Minoshima ${ }^{2}$, Farah Younus ${ }^{4}$, \\ S. Qasim Mehdi' ${ }^{4}$, Uppala Radhakrishna ${ }^{1}$, Marie-Pierre Papasavvas ${ }^{1}$, Corinne Gehrig ${ }^{1}$, Colette Rossier ${ }^{1}$, Michael \\ Korostishevsky ${ }^{3}$, Andreas Gal ${ }^{5}$, Nobuyoshi Shimizu ${ }^{2}$, Batsheva Bonne-Tamir ${ }^{3} \&$ Stylianos E. Antonarakis ${ }^{1}$
}

\begin{abstract}
Approximately $\mathbf{5 0 \%}$ of childhood deafness is caused by mutations in specific genes. Autosomal recessive loci account for approximately $\mathbf{8 0} \%$ of nonsyndromic genetic deafness ${ }^{1}$. Here we report the identification of a new transmembrane serine protease (TMPRSS3; also know $\mathbf{n}$ as ECHOS1) expressed in many tissues, including fetal cochlea, which is mutated in the families used to describe both the DFNB10 and DFNB8 loci. An 8-bp deletion and insertion of 18 monomeric ( 68-bp) $\beta$-satellite repeat units, normally present in tandem arrays of up to several hundred kilobases on the short arms of acrocentric chromosomes, causes congenital deafness (DFNB10). A mutation in a splice-
\end{abstract}

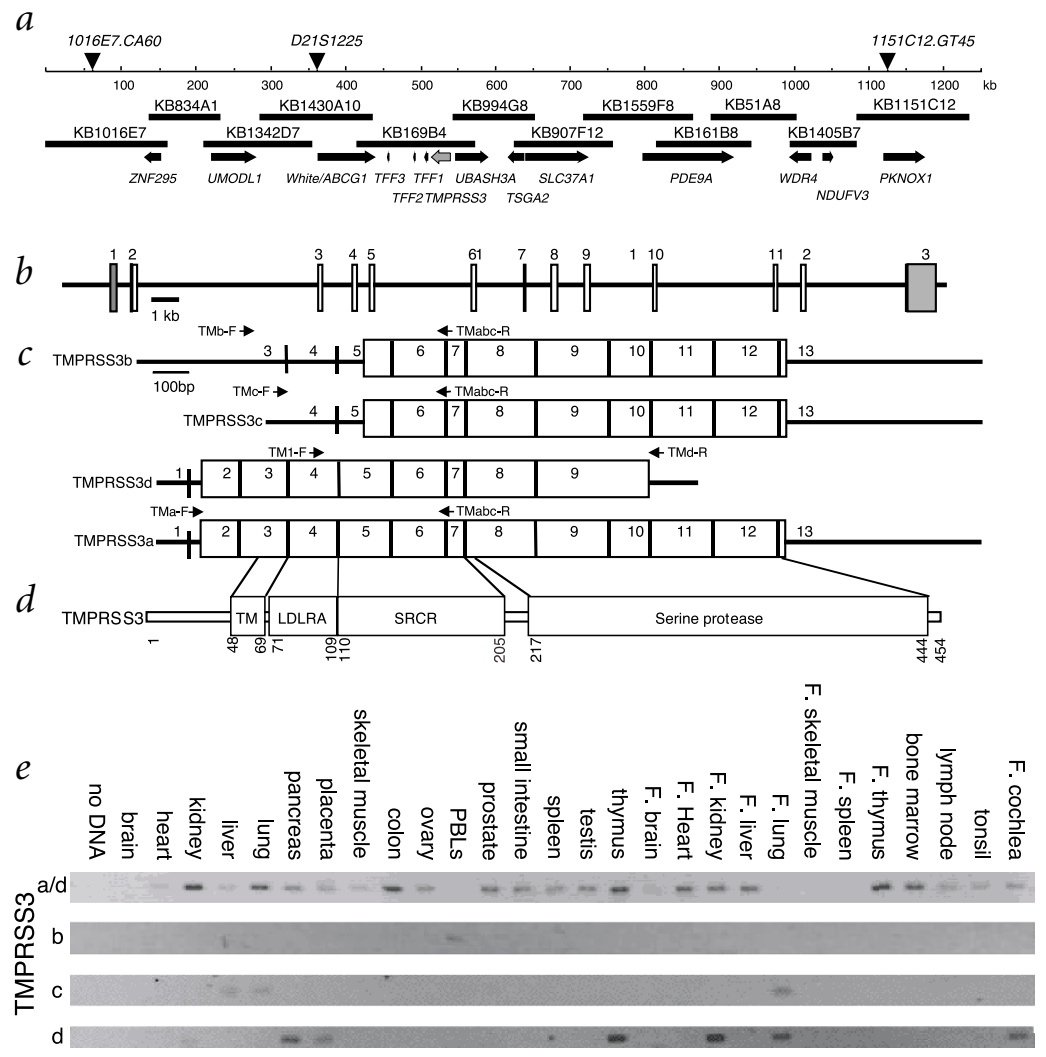

acceptor site, resulting in a 4-bp insertion in the mRNA and a frameshift, was detected in childhood onset deafness (DFNB8). This is the first description of $\beta$-satellite insertion into an active gene resulting in a pathogenic state, and the first description of a protease involved in hearing loss.

Two families with autosomal recessive sensorineural nonsyndromic deafness were previously independently reported with linkage to distal chromosome 21q. A locus in a Pakistani family was mapped telomeric to D21S1225 in 21q22.3 (DFNB8; refs. 2,3), whereas the locus in a Palestinian family ${ }^{4}$ mapped to an approximately $1-\mathrm{Mb}$ region between markers 1016E7.CA60 and 1151C12.GT45 (DFNB10; ref. 5). We previously reported the exclusion of nine genes in the DFNB10 critical region ${ }^{5-8}$ and the exclusion of three candidate genes (telomeric to the DFNB10 critical region) in the DFNB8 critical region ${ }^{3}$.

Further analysis of the DFNB10 critical region ${ }^{9}$ indicated the presence of four additional genes (ZNF295, UMODL1, TMPRSS3 and TSGA2; Fig. 1a), including a new gene, TMPRSS3 (for transmembrane protease, serine 3), with 13 exons spanning $24 \mathrm{~kb}$ (Fig. $1 b$; for exon-intron junctions, see Table A, http:// genetics.nature.com/supplementary_info/). We detected four alternative transcripts, TMPRSS3a (2,438 bp), TMPRSS3b (2,524

Fig. 1 The DFNB10 critical region and TMPRSS3. a, A map of the DFNB10 critical region in chromosome 21q22.3. The positions of the microsatellite markers defining the DFNB10 and DFNB8 critical region in $21 q 22.3$ are shown. The BAC contig between markers 1016E7.CA60 and 1151C12.GT45 is shown, with six known genes (ABCG1, TFF3, TFF2, TFF1, PDE9A NDUVF3) and seven novel genes (ZNF295, UMODL1, TMPRSS3, UBASH3A, TSGA2, SLC37A1, WDR4) mapping to the critical region. b, TMPRSS3 contains 13 exons (boxes) spanning $24 \mathrm{~kb}$. c, There are four different transcripts, TMPRSS3a-d (coding regions in boxes noncoding regions indicated by narrow box). d, A schematic of the TMPRSS3 protein showing the transmembrane (TM), LDLRA, SRCR and protease domains and their position in the 454-aa peptide. e, Expression analysis of the TMPRSS3 transcripts. RT-PCR specific to the TMPRSS3a- $d$ transcripts were performed on CDNA from 27 human tissues and fetal cochlea as indicated.

${ }^{1}$ Division of Medical Genetics, University of Geneva Medical School, Genève, Switzerland. ${ }^{2}$ Department of Molecular Biology, Keio University School of Medicine, Shinjuku-ku, Tokyo, Japan. ${ }^{3}$ Department of Human Genetics and Molecular Medicine, Sackler School of Medicine, Tel-Aviv University, Ramat-Aviv, Israel. ${ }^{4}$ Biomedical and Genetic Engineering Division, Khan Research Laboratories, Islamabad, Pakistan. ${ }^{5}$ Institut fur Humangenetik, Universitats-Krankenhaus Eppendorf, Hamburg, Germany. ${ }^{6}$ Present address: Genetics and Bioinformatics Division, Walter and Eliza Hall Institute, Royal Parade, Parkville, P.O. Royal Melbourne Hospital, Victoria, Australia. Correspondence should be addressed to S.E.A. (e-mail: Stylianos.Antonarakis@medecine.unige.ch). 
bp), TMPRSS3c (2,105 bp) and TMPRSS3d (1,359 bp), encoding putative polypeptides of 454 , 327, 327 (same as TMPRSS3b) and 344 amino acids, respectively. The TMPRSS3a transcript contains all 13 exons with the initiating methionine codon in exon 2. The TMPRSS3b and TMPRSS $3 c$ transcripts start in introns 2 and 3 , respectively, with putative initiating methionine codons in exon 5 . The $3^{\prime}$

end of TMPRSS3d is derived from 5 ESTs (for example AI978874) and continues into intron 9 (Fig. 1c).

Northern-blot analysis showed weak expression of the 2.4- and 1.4-kb TMPRSS3a and TMPRSS3d transcripts (data not shown). Semi-quantitative RT-PCR revealed that all four transcripts show distinct patterns of expression, but TMPRSS3a is the most abundantly and widely expressed transcript, including in fetal cochlea (Fig. 1e).

The putative peptide encoded by TMPRSS3a contains transmembrane (TM), low-density-lipoprotein receptor A (LDLRA), scavenger-receptor cysteine-rich (SRCR) and serine protease domains (Fig. 1d) similar to other proteases, including that encoded by the homologous gene TMPRSS2, which also maps on

$a$

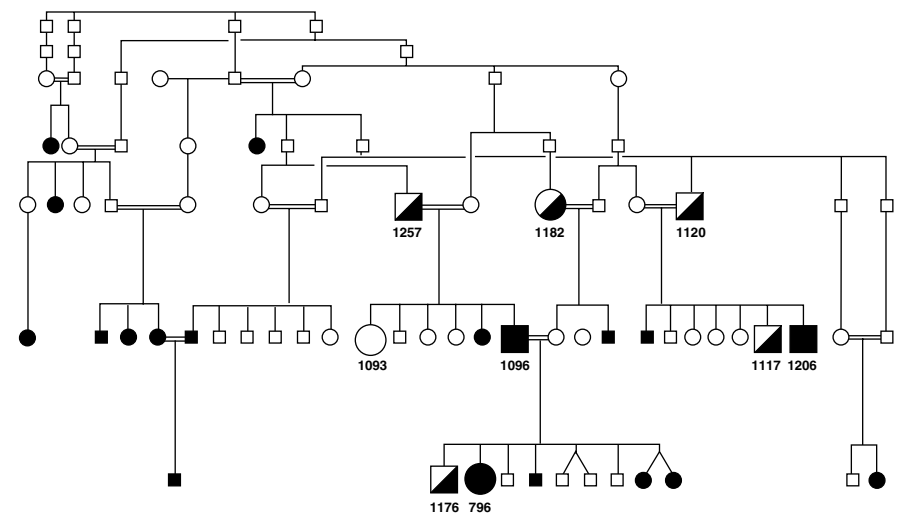

c

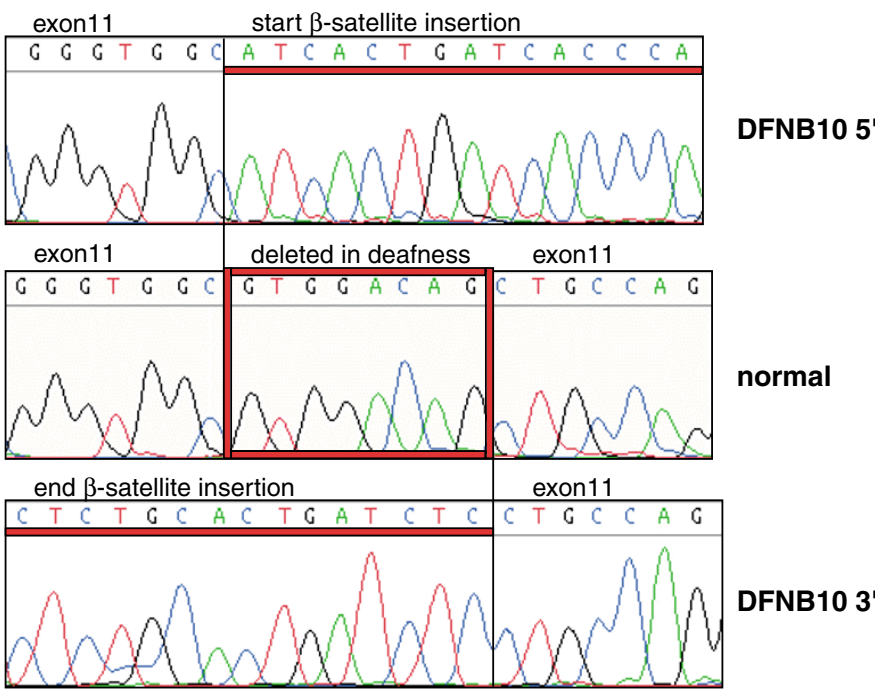

21q, centromeric to the DFNB10 critical region ${ }^{10}$. The serine protease domain (residues 217-444) is compatible with the S1 family of the PA clan of serine-type peptidases (serine or cysteine nucleophile; catalytic residues in the order His, Asp, Ser (or Cys) in sequence; all endopeptidases) for which the prototype is chymotrypsin ${ }^{11}$, and shows between 45 and $38 \%$ identity with other transmembrane serine proteases (Fig. $1 f$ ).

As no recognizable leader sequence precedes the predicted hydrophobic TM domain, TMPRSS3 is likely to be a type II integral membrane protein with a cytosolic amino terminus and an extracellular protease domain, similar to other transmembrane proteases ${ }^{12}$. The LDLRA domain, which contains six disulfidebound cysteines (C72, C79, C85, C92, C98 and C107), was originally found in the low density lipoprotein receptor as the binding site for low-density lipoprotein and calcium ${ }^{13}$, and has subsequently been described in numerous extracellular and membrane proteins. SRCR domains linked to serine protease domains have been reported in secreted or membrane-bound molecules with diverse biological roles in development and immunity ${ }^{14}$. The LDLRA and SRCR domains of TMPRSS3 may be involved in binding with extracellular molecules or the cell surface.

The putative peptides encoded by the TMPRSS $3 b$ and TMPRSS3c transcripts contain only half of the SRCR domain, whereas the

$b$

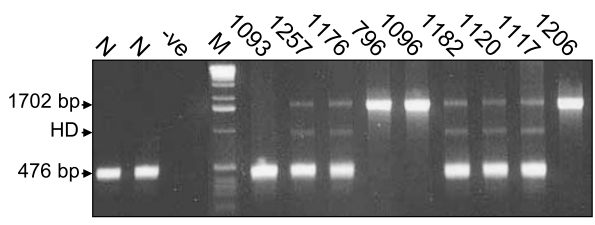

Fig. 2 Identification of a $\beta$-satellite insertion in exon 11 of TMPRSS3. a, The Palestinian DFNB10 pedigree is shown with homozygous affected and heterozygous individuals marked by filled and half-filled symbols, respectively. b, PCR amplification of exon 11 of TMPRSS3 from selected individuals (labeled in a and indicated by larger symbols) shows inheritance of the 1702-bp band with deafness. $\mathrm{N}$, hearing control; -ve, no DNA control; $M$, marker. The position of the normal 476-bp, mutant 1702 and heteroduplex (HD) bands seen in heterozygotes are indicated (arrow pointing to the left). c, A chromatogram from a normal individual is shown aligned with chromatograms showing the $5^{\prime}$ (DFNB10 5) and 3 (DFNB10 3) sequences of the insertion. $\beta$-satellite sequences specific to the chromatograms from affected individuals are underlined in red and the $8 \mathrm{nt}$ deleted at the point of insertion are boxed in red in the normal chromatogram. This would result in a frameshift mutation from $\mathrm{G} 393$ within the protease domain of TMPRSS3 and termination at 404 amino acids after the addition of 11 unrelated amino acids. 
Fig. 3 Homology of the $\beta$-satellite monomers and FISH analysis. a, An alignment of the $18 \beta$-satellite monomers inserted into exon 11 of TMPRSS3 shows the high homology of the monomeric subunits. b. Two-color FISH analysis of chromosomes 21 from homozygous affected individuals from the DFNB10 family was performed using a BAC containing TMPRSS3 (KB169B4, red) and a $\beta$ satellite probe generated by PCR from the insertion (green). The overlapping green and red probes produce yellow. c, Homology between two sections of exon 11 of TMPRSS3 (shaded gray) and the $\beta$-satellite repeats results in the recombination and subsequent insertion of $18 \beta$ satellite repeats at the position marked by an arrow. d, The homology of the second block, 74 nucleotides of exon 11 of TMPRSS3 just before the point of insertion, with a sequence-spanning repeats 10 and 11 of the insertion.

putative product of TMPRSS3d contains only half of the protease domain (Fig. 1c). The TMPRSS3b and TMPRSS $3 c$ transcripts may be artifacts of RACE experiments or may produce soluble forms of the protease as has been observed for hepsin ${ }^{15}$.

Direct sequencing of all 13 exons of TMPRSS3 (PCR amplified from DNA samples from the Palestinian DFNB10 and Pakistani DFNB8 families ${ }^{2,4,5}$ and controls) detected two sequence variants in the Palestinian/DFNB10 family (changes 4 and 5; Table 1). Amplification of exon 11 of TMPRSS3 from a DFNB10 sample resulted in a 1.7-kb product instead of the 476bp product that was amplified from normal controls. Southernblot and PCR-assay analyses of exon 11 of TMPRSS3 showed that the size of the insertion does not vary between family members or generations (data not shown), unlike fragile sites and repeatexpansion disorders ${ }^{16}$. Eight affected individuals were homozygous for the $1.7-\mathrm{kb}$ product and 13 obligate carriers had one copy. The insertion segregated with the phenotype (Fig. 2a,b). Sequence analysis revealed a complex rearrangement with the deletion of $8 \mathrm{bp}$ and the insertion of 18 complete $\beta$-satellite repeat monomers with 10 and 2 bp derived from $\beta$-satellite repeats at the $5^{\prime}$ and $3^{\prime}$ ends of the rearrangement, respectively (Fig. $2 c$ ). The sequences of the $18 \beta$-satellite monomers, although highly conserved, are also highly variable (52-93\% divergence between repeats; Fig. 3a). Monomeric, approximately 68-bp repetitive units of $\beta$-satellites (or Sau3A repeats) have been detected on the short arms of all human acrocentric chromosomes and chromosomes 1, 9 (centromeric), 19p and Y (refs. $17-19)$. At least four families of $\beta$-satellites have been defined ${ }^{19}$ and the $\beta$-satellite insertion into TMPRSS3 is likely to be derived from either $\mathrm{p} 21 \beta 2$ or $\mathrm{p} 21 \beta 7$ repeats.

Using two-color FISH, we detected TMPRSS3 signal only on chromosome $21 \mathrm{q} 22.3$, and $\beta$-satellites at the described localizations in normal chromosomes (data not shown). Colocalization was observed at $21 \mathrm{q} 22.3$ on chromosomes 21 derived from individuals heterozygous and homozygous for the insertion (Fig. $3 b$ ).

The mobile nature of repetitive sequences on the short arms of acrocentric chromosomes is well documented ${ }^{20}$. Circular extrachromosomal molecules present in many eukaryotic cells, small polydisperse circular DNAs (spcDNA), may contain $\beta$ satellites ${ }^{21}$ or other repeats present on the short arms of human acrocentric chromosomes (for review, see ref. 22), and are probably produced by unequal homologous recombination between or within repetitive sequences. The insertion into TMPRSS3 in the DFNB10 family may have arisen by recombination of spcDNA containing $\beta$-satellites with a region of minimal homology spanning exon 11 of TMPRSS3 (Fig. $3 c, d$ ). Although chromosomal rearrangements involving $\beta$-satellites have been described $^{23,24}$, this is the first description of $\beta$-satellite insertion into an active gene resulting in a pathogenic state.

Three TMPRSS3 sequence variants were observed in homozygosity in the Pakistani/DFNB8 family ${ }^{2}$ (changes 1, 2 and 6; Table 1). A G $\rightarrow$ A substitution at IVS4-6, whose inheritance was consistent with deafness in branches 3 and 4 of the DFNB8 family, creates an alternative splice-acceptor site (Fig. $4 a, b$ ) and was observed in 1 of 160 Muslim Indians and none of 30 European chromosomes. Haplotype analysis on the heterozygous, hearing individual with IVS4-6G $\rightarrow \mathrm{A}$, and his/her spouse and child, with 4 intragenic TMPRSS3 SNPs (changes 1, 3, 4 and 6; Table 1) was consistent with a single mutational origin of IVS4-6G $\rightarrow$ A.

We next assessed the effect of IVS4-6G $\rightarrow$ A on splicing in vitro (Fig. 4b). RT-PCR and sequencing revealed a 4-bp insertion between exons 4 and 5 (Fig. $4 c, d$ ), consistent with the use of the putative splice acceptor site created by IVS4-6G $\rightarrow$ A. We screened 179 cloned RT-PCR products from an affected individual with an oligonucleotide that mimics the normal splice junction and one that includes the insertion. We found no hybridization with the 'normal' probe. Thus IVS4-6G $\rightarrow$ A can be considered to be a pathogenic mutation. Mutations in spliceacceptor sites that allow some normal splicing often result in 
$a$

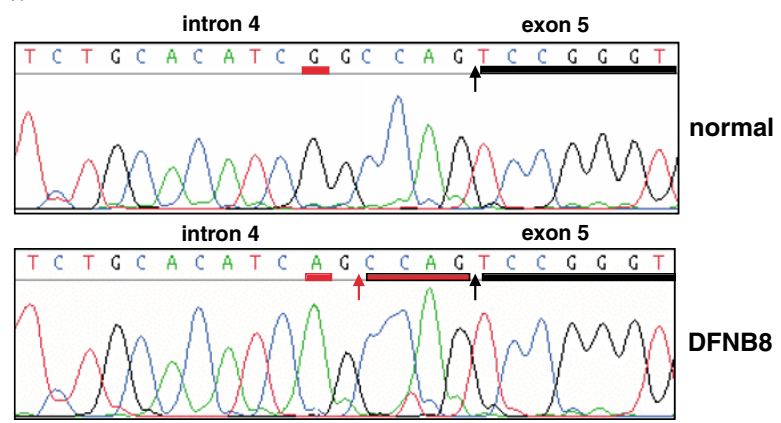

Fig. 4 Identification of a splice-site mutation in TMPRSS3 in the Pakistani DFNB8 family. a, Chromatograms of normal and affected individuals from the DFNB8 family showing the $\mathrm{G} \rightarrow \mathrm{A}$ substitution at IVS4-6 underlined in red. The normal intron 4 splice-acceptor site (black arrow) and the putative acceptor site created by IVS4-6G $\rightarrow$ A (red arrow) are indicated. b, A genomic fragment containing exons 4 and 5 of TMPRSS3 was inserted into an

exon trapping (splicing) vector. c,d, RT-PCR from RNA of transfected COS7 cells identified a 4-bp insertion (underlined in red in d) between exons 4 and 5 (b-d) consistent with the use of the putative splice acceptor site created by IVS4-6G $\rightarrow$ A. The 4-bp insertion would result in a frameshift from C107, and termination at 132 aa after the addition of 25 unrelated amino acids.

milder phenotypes compared with null mutations in the same gene $^{25}$. In vivo, the IVS4-6G $\rightarrow$ A mutation in the DFNB8 family may allow limited normal splicing, and thus some normal TMPRSS3 protein, accounting for the phenotypic difference between the DFNB8 and DFNB10 families.

Many human disorders such as hereditary pancreatitis (PRSS1, MIM 167800) and coagulation factor deficiencies (factors VII, IX, X and XII) are due to deficiency of serine proteases. This, however, is the first description of a protease involved in hearing loss. The phenotypic differences between the two families studied here may imply that TMPRSS3 polymorphisms resulting in or associated with reduced expression or slightly abnormal function might be involved in age-related hearing loss, the most common form of hearing loss. The sensorineural hearing loss in both families with TMPRSS3 mutations implies defects in the inner ear. TMPRSS3 expression was demonstrated in fetal cochlea and many other tissues. TMPRSS3 may be involved in the development and maintenance of the inner ear or the contents of the perilymph and endolymph. The levels of total protein in endolymph are extremely low, but endolymph proteins show a prominence of glycosylated acidic proteins and turnover of these proteins may require specific proteases ${ }^{26}$.

\section{Methods}

Sample collection. The DFNB10 family (BT117) is a large, inbred, Palestinian family from a small town in Israel ${ }^{4}$. Pure-tone audiometric tests showed profound hearing loss in all affected individuals, without any hearing remnants, at a level of 75-80 decibels. Sensorineural deafness was confirmed in one-week-old twin girls by a brain stem-evoked potential (BERA) test. Blood samples were obtained from 36 hearing and 16 deaf individuals after obtaining informed consent and in accordance with institutional guidelines for human subjects.

The DFNB8 family is a large, consanguineous kindred from Pakistan ${ }^{2}$. Pure-tone audiometric tests, between 125 and $8,000 \mathrm{~Hz}$ up to $120 \mathrm{~dB}$, revealed a maximum audio threshold in both ears of affected individuals of $105 \mathrm{~dB}$ at $1,000 \mathrm{~Hz}$. Onset of hearing-related problems was between 10 and $12 \mathrm{y}$ of age $b$

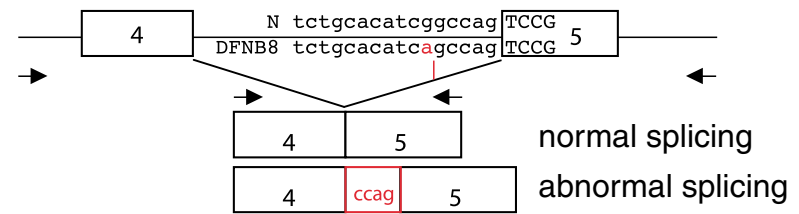

$d$

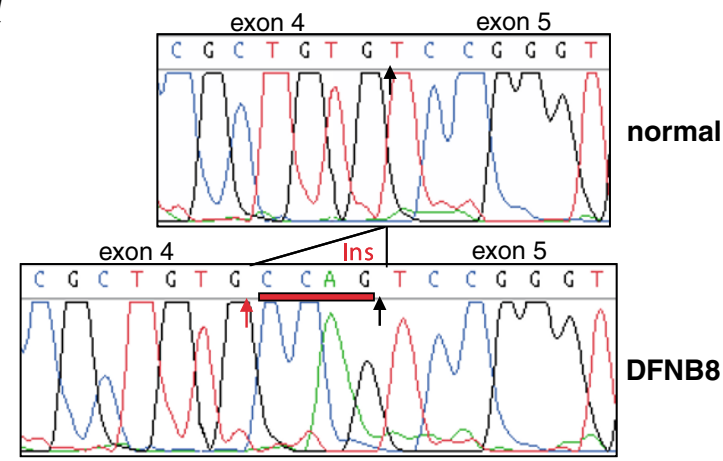

with profound hearing loss evident at 14 to $16 \mathrm{y}$. We obtained blood samples from 16 hearing and 8 deaf individuals after obtaining informed consent and in accordance with institutional guidelines for human subjects.

Bacterial clone contigs, genomic sequencing and isolation of TMPRSS3 cDNAs. A BAC/cosmid contig spanning MX1-D21S171 was constructed (see http://www.dmb.med.keio.ac.jp/seqpub/map/APECED.html). Genomic sequencing and analysis was as described ${ }^{9}$, and primers were designed to predicted exons (Table B, see http://genetics.nature.com/supplementary_info/). We amplified TMPRSS3 cDNA fragments with primer pairs TM1-F/TM1-R and TM2-F/TM2-R. We carried out 5'- and 3'-RACE with primer pairs TMRA-R1/AP1, TMRA-R2/AP2, TMRA-F1/AP1 and TMRA-F2/AP2. RT-PCR was carried out on thymus, spleen, lymph node, liver and placenta cDNA pools (MTC cDNA and Marathon-Ready cDNA, Clontech) using 35 cycles of $94^{\circ} \mathrm{C}$ for $30 \mathrm{~s}, 65^{\circ} \mathrm{C}$ for $1 \mathrm{~min}$, and $72^{\circ} \mathrm{C}$ for $2 \mathrm{~min}$. PCR products were cloned into pBluescript II(SK+) (Stratagene) and sequenced. The sequence of predicted proteins of TMPRSS3 were analyzed using web-based tools including TMPRED (transmembrane prediction; http://ulrec3. unil.ch/software/TMPRED_form.html), and domain searches using the Pfam databases (http://www.sanger.ac.uk/Software/Pfam/search.shtml).

Multiple-tissue cDNA panel and northern-blot analysis. Expression analysis of TMPRSS3 was performed using the Human Multiple Tissue cDNA (MTC) panels (Clontech; I, II, fetal, and immune system panels) containing cDNAs from 27 human tissues and a cDNA prepared from fetal cochlea (18 weeks) mRNA. TMPRSS3 cDNAs were amplified with primer pairs TMa-F/TMabc$\mathrm{R}$ (TMPRSS3a), TMb-F/TMabc-R (TMPRSS3b), TMc-F/TMabc- $\mathrm{R}$ (TMPRSS3c) and TM1-F/TMd-R (TMPRSS3d) using 20 pg of each cDNA as template, whereas the control PCR (a G3PDH amplimer) was amplified from $2 \mathrm{pg}$ of cDNA. Northern blots containing $2 \mu \mathrm{g}$ poly $(\mathrm{A})^{+} \mathrm{RNA}$ from 23 different adult human tissues (Clontech Human MTN Blots 1-3) were hybridized with a ${ }^{32}$ P-labeled probe (nt 1-517 of TMPRSS3a).

Mutation analyses. We determined the genomic structure of TMPRSS3 by comparing the genomic sequence with the cDNA sequences. Each TMPRSS3 exon and each flanking regions were amplified from patient and unrelated control DNAs (for primers see Table C, http://genetics.nature. com/supplementary_info/) and analysed as described ${ }^{6}$. PCR products 
containing exons 4 and 5 were amplified from a DFNB8 and a normal sample and the fragments were cloned into the exon-trapping vector pSPL3 (Gibco-BRL). Transfections, RNA isolation and RT-PCR were performed as described ${ }^{27}$ between oligonucleotides 7614-TMP4eF (5'-GCT CATCCTTTAAGTGTATCG-3') and 7615-TMP5eR (5'-ATGGTCTTCCAC GAAGCAGCTG-3'). Products were cloned into pTOPO (Invitrogen) and the 4-nt insertion was assayed by colony hybridization with oligonucleotides 7737-E4E5con (5'-AGTACCGCTGTGTCCGGGT-3', normal) and 7738E4E5deaf (5'-CCGCTGTGCCAGTCCGGGT-3', mutant 4-nt underlined).

FISH analyses. BAC KB169B4 (containing TMPRSS3) and the mutant exon 11 PCR product containing $18 \beta$-satellite repeats were labeled using the Biotin and DIG-nick translation mixes (Roche), respectively, and detected with Alexa Fluor 568 conjugate of Streptavidin (Molecular Probes) and sheep-anti-digoxigenin-fluorescin antibodies (Roche), respectively. Metaphase spreads were prepared using cultured lymphoblastic cells (from normal, heterozygote and affected individuals) and hybridized with a mixture of both probes using ChromaHyb 600 hybridization buffer (Quantum Biotechnologies).

GenBank accession numbers. Genomic sequence of BAC KB169B4, AP001623; human TMPRSS3a, TMPRSS3b, TMPRSS3c and TMPRSS3d cDNAs, AB038157, AB038158, AB038159 and AB038160, respectively.

\section{Acknow ledgments}

We thank S. Dahoun, C. Vieux and M.A. Morris for help with the FISH analyses; all members of the S.E.A. laboratory, past and present, for transcription mapping; and A. Shintani, T. Sasaki, K. Nagamine, M. Takahashi, M. Sasaki and all members of the genomic sequencing team in the Laboratory of Genomic Medicine, Keio University School of Medicine for their contribution to this work. The laboratory of S.E.A. is supported by grants from the Swiss FNRS, the OFES/EU, and funds from the University and Cantonal Hospital of Geneva. The laboratory of B.B.-T. is supported in part by grants from the Applebaum Foundation. The Laboratory of Genomic Medicine, Keio University School of Medicine was supported in part by a Fund for Human Genome Sequencing Project from the Japan Science and Technology Corporation; Grants in Aid for Scientific Research on Priority Areas from the Ministry of Education, Science, Sports and Culture of Japan; and Grants in Aid for Scientific Research and a Fund for "Research for the Future" Program from the Japan Society for the Promotion of Science. The laboratory of A.G. is supported in part by grants from the FAUN-Stiftung.

\section{Received 8 September; accepted 28 November 2000.}

1. Kalatzis, V. \& Petit, C. The fundamental and medical impacts of recent progress in research on hereditary hearing loss. Hum. Mol. Genet. 7, 1589-1597 (1998).

2. Veske, A. et al. Autosomal recessive non-syndromic deafness locus (DFNB8) maps on chromosome 21q22 in a large consanguineous kindred from Pakistan. Hum Mol. Genet. 5, 165-168 (1996).

3. Scott, H.S. et al. Refined genetic mapping of the autosomal recessive non- syndromic deafness locus DFNB8 on human chromosome 21q22.3. Adv. Otorhinolaryngol. 56, 158-163 (2000).

4. Bonne-Tamir, B. et al. Linkage of congenital recessive deafness (gene DFNB10) to chromosome 21q22.3. Am. J. Hum. Genet. 58, 1254-1259 (1996).

5. Berry, A. et al. Refined localization of autosomal recessive nonsyndromic deafness DFNB10 locus using 34 novel microsatellite markers, genomic structure, and exclusion of six known genes in the region. Genomics 68, 22-29 (2000).

6. Michaud, J. et al. Isolation and characterization of a human chromosome 21q22.3 gene (WDR4) and its mouse homologue that code for a WD-repeat protein. Genomics 68, 71-79 (2000).

7. Bartoloni, L. et al. Cloning and characterization of a putative human glycerol 3 phosphate permease gene (SLC37A1 or G3PP) on 21q22.3: mutation analysis in 2 candidate phenotypes, DFNB10 and a glycerol kinase deficiency. Genomics 70 190-200 (2000).

8. Wattenhofer, $\mathrm{M}$. et al. Isolation and characterization of the UBASH3A gene on 21q22.3 encoding a potential nuclear protein with a novel combination of domains: mutation analysis in DFNB10. Hum. Genet. (in press).

9. The Chromosome 21 Mapping and Sequencing Consortium. The DNA sequence of human chromosome 21. Nature 405, 311-319 (2000).

10. Paoloni-Giacobino, A., Chen, H., Peitsch, M.C., Rossier, C. \& Antonarakis, S.E. Cloning of the TMPRSS2 gene, which encodes a novel serine protease with transmembrane, LDLRA, and SRCR domains and maps to 21q22.3. Genomics 44 309-320 (1997).

11. Rawlings, N.D. \& Barrett, A.J. Families of serine peptidases. Methods Enzymol. 244, 19-61 (1994).

12. Tsuji, A. et al. Hepsin, a cell membrane-associated protease. Characterization tissue distribution, and gene localization. J. Biol. Chem. 266, 16948-16953 (1991).

13. van Driel I R. Goldstein, J.L, Sudhof T.C. \& Brown, M.S. First cysteine-rich repeat in ligand-binding domain of low density lipoprotein receptor binds $\mathrm{Ca}^{2+}$ and monoclonal antibodies, but not lipoproteins. J. Biol. Chem. 262, 17443-17449 (1987).

14. Resnick, D., Pearson, A. \& Krieger, M. The SRCR superfamily: a family reminiscent of the Ig superfamily. Trends Biochem. Sci. 19, 5-8 (1994).

15. Kawamura, S., Kurachi, S., Deyashiki, Y. \& Kurachi, K. Complete nucleotide sequence, origin of isoform and functional characterization of the mouse hepsin gene. Eur. J. Biochem. 262, 755-764 (1999).

16. Handt, O., Sutherland, G.R. \& Richards, R.I. Fragile sites and minisatellite repeat instability. Mol. Genet. Metab. 70, 99-105 (2000).

17. Greig, G.M. \& Willard, H.F. $\beta$ satellite DNA: characterization and localization of two subfamilies from the distal and proximal short arms of the human acrocentric chromosomes. Genomics 12, 573-580 (1992).

18. Eichler, E.E. et al. Complex $\beta$-satellite repeat structures and the expansion of the zinc finger gene cluster in 19p12. Genome Res. 8, 791-808 (1998).

19. Shiels, C., Coutelle, C. \& Huxley, C. Contiguous arrays of satellites 1 , 3 , and $\beta$ form a 1.5-Mb domain on chromosome 22p. Genomics 44, 35-44 (1997).

20. Farrell, S.A., Winsor, E.J. \& Markovic, V.D. Moving satellites and unstable chromosome translocations: clinical and cytogenetic implications. Am. J. Med. Genet. 46, 715-720 (1993).

21. Assum, G., Fink, T., Steinbeisser, T. \& Fisel, K.J. Analysis of human extrachromosomal DNA elements originating from different $\beta$-satellite subfamilies. Hum. Genet. 91, 489-495 (1993).

22. Gaubatz, J.W. Extrachromosomal circular DNAs and genomic sequence plasticity in eukaryotic cells. Mutat. Res. 237, 271-292 (1990).

23. Wolff, D.J. \& Schwartz, S. Characterization of Robertsonian translocations by using fluorescence in situ hybridization. Am. J. Hum. Genet. 50, 174-181 (1992).

24. Samonte, R.V., Conte, R.A., Ramesh, K.H. \& Verma, R.S. Molecular cytogenetic characterization of breakpoints involving pericentric inversions of human chromosome 9. Hum. Genet. 98, 576-580 (1996).

25. Scott, H.S. et al. Identification of mutations in the $\alpha$-L-iduronidase gene (IDUA) that cause Hurler and Scheie syndromes. Am. J. Hum. Genet. 53, 973-986 (1993).

26. Thalmann, R. \& Thalmann, I. Source and role of endolymph macromolecules. Acta Otolaryngol. 119, 293-296 (1999).

27. Chen, $\mathrm{H}$. et al. Cloning of 559 potential exons of genes of human chromosome 21 by exon trapping. Genome Res. 6, 747-760 (1996).

28. Dunnen, J.T. \& Antonarakis, S.E. Mutation nomenclature extensions and suggestions to describe complex mutations: a discussion. Hum. Mutat. 15, 7-12 (2000) 